\title{
Corrosion resistance studies of carbon-encapsulated iron nanoparticles
}

\author{
Maciej Fronczak ${ }^{1}$ (1), Olga Łabędź ${ }^{1}$, Waldemar Kaszuwara ${ }^{2}$, and Michał Bystrzejewski ${ }^{1, *}$ (1) \\ ${ }^{1}$ Faculty of Chemistry, University of Warsaw, Pasteur 1 Street, 02-093 Warsaw, Poland \\ ${ }^{2}$ Faculty of Materials Science, Warsaw University of Technology, Wołoska 141 Street, 02-507 Warsaw, Poland
}

Received: 19 September 2017

Accepted: 2 November 2017

Published online:

13 November 2017

(C) The Author(s) 2017. This article is an open access publication

\begin{abstract}
The carbon coating in carbon-encapsulated magnetic nanoparticles is considered as a tight and impermeable barrier which should perfectly protect the magnetic core material from external chemical environment. To study the integrity of the coating, carbon-encapsulated iron nanoparticles were subjected to corrosion tests, in which various corrosion agents were used. Several mineral and organic acids, as well as active gaseous environments with various oxidation potential, were applied. Additionally, the corrosion resistance was studied under the so-called galvanic corrosion, using two metal ions (copper and silver) which have higher redox potential than the zero-valent iron. The release of iron from the core as well as the morphology, structural features, chemical composition, and magnetic properties of carbon-encapsulated iron nanoparticles was systematically monitored at each stage of the corrosion process. The largest release of Fe from the encapsulate core was observed when nitric acid was used as the corrosion agent.
\end{abstract}

\section{Introduction}

Carbon-encapsulated nanoparticles were discovered in 1993 [1] during the intensive research on the formation of fullerenes and carbon nanotubes in carbon arc discharge. The pioneering work by Ruoff et al. showed that the encapsulation of hydroscopic $\mathrm{LaC}_{2}$ in a protective carbon shell significantly improved its stability in contact with ambient humidity. The first report on carbon-encapsulated magnetic nanoparticles (containing iron, nickel or cobalt) was published in 1994 [2]. Since that time hundreds of papers on the synthesis and applications of carbon-encapsulated nanoparticles have been published. A quite actual summary on carbon encapsulates, primarily containing magnetic nanoparticles, can be found in the following papers and reviews [3-6].

The carbon coating in carbon encapsulates has three main features: (1) it stabilizes and protects the encapsulated nanoparticles from the ambient environment, (2) minimizes the interaction between the adjacent encapsulates, and (3) is a suitable scaffold for further functionalization [7-11]. The first feature is the most important one because the properly

Address correspondence to E-mail: mibys@chem.uw.edu.pl 
developed carbon coating can be a perfect barrier which can sufficiently protect the core material against corrosion. The corrosion resistance of carbon encapsulates is of great importance, since many surface functionalization methods involves the use of different acids, e.g., nitric acid [9]. Only a few reports which deal with the corrosion resistance of carbonencapsulated magnetic nanoparticles have been published. Taylor et al. studied the influence of acid treatment (hydrochloric acid, nitric acid, sulfuric acid and their mixtures) on the morphology and magnetic properties of carbon-encapsulated iron nanoparticles [12]. They have found that the treatment in a mixture of $\mathrm{H}_{2} \mathrm{SO}_{4} / \mathrm{HNO}_{3}$ causes the greatest mass loss and the highest decrease of magnetization. Similar findings come from the paper by Schumacher et al., which described that concentrated aqua regia results in severe perforation of carbon coatings and leads to the dissolution of pristinely encapsulated magnetic nanoparticles [13]. Furthermore, Fernandez-Garcia et al. [14] studied the stability of carbon-coated nickel nanoparticles embedded in the pores of mesoporous carbon matrix. They demonstrated that $0.1 \mathrm{M} \mathrm{HCl}$ does not etch the magnetic phase. Moreover, they observed that carbon coating perfectly protected the nickel core from the aerial oxidation even for 36 months. Similarly, Schwickardi et al. [15] studied carbon-coated iron nanoparticles surrounded by activated carbon and obtained similar results to paper [14]. The work of Haslam et al. [16] also confirmed that thin films formed of nickel nanocrystals encapsulated in carbon had well corrosion stability in hot $1.5 \mathrm{M}$ sulfuric acid. The stability was also observed when the thin film worked as the electrode which was electrically polarized. The importance of the corrosion studies also results from the prospective applications of nanomaterials which contain iron nanoparticles. Iron nanoparticles, because of their magnetic performance, can be used for the development of mobile composite adsorbents. It has been recently shown that the composite of CEINs with porous carbon matrix obtained from waste PET efficiently removes phenolic compounds from aqueous solutions [17]. In that application high corrosion resistance is needed to avoid any unwanted chemical processes between the encapsulated iron nanoparticles and the species of the solution. Furthermore, the corrosion resistance of carbon-encapsulated iron nanoparticles is also important when they are applied as a catalyst, e.g., in oxygen reduction [18]. There are many other possibilities to use iron-containing nanomaterials, where high corrosion stability is desired. The possible applications of such nanomaterials are described in the following reviews [19, 20], and they cover remediation of heavy metal ions, inorganic anions, water disinfection, magnetic-guided drug delivery systems, magnetic resonance imaging, and magnetic hyperthermia.

In this paper we present very systematic studies of the corrosion resistance of carbon-encapsulated iron nanoparticles. We have tested the corrosion resistance in the presence of a broad range of corrosion agents, which include a series of cool and boiled mineral and organic acids, metal cations with high redox potential, and ionized gases.

\section{Experimental}

Carbon-encapsulated iron nanoparticles were synthesized in a carbon arc discharge reactor. The detailed procedure is described elsewhere [21]. In brief, graphite rods ( $8 \mathrm{~mm}$ in diameter) were used as cathodes. The anodes were made of drilled graphite rods, which were filled with a mixture of fine graphite and iron powders. The total Fe content in the anode was $45 \mathrm{wt} \%$. The Fe-doped anodes were sublimated under $\mathrm{Ar}-\mathrm{H}_{2}$ (1:1) atmosphere at the pressure of $60 \mathrm{kPa}$.

The raw product ( $\mathrm{r}$-CEINs) contains both the nonencapsulated iron nanoparticles and the nanoparticles which are encapsulated in carbon coatings. The purified carbon-encapsulated iron nanoparticles ( $p$ CEINs) were obtained after purification, in which the raw product was refluxed for $24 \mathrm{~h}$ in $3 \mathrm{M}$ hydrochloric acid (with subsequent washing in excess of water and ethanol). The purification yield was $61 \pm 2 \mathrm{wt} \%$. (This range corresponds to five purification tests.) The corrosion resistance studies in acids were conducted at room and boiling temperature. Three hundred milligrams of p-CEINs was added to $250 \mathrm{~cm}^{3}$ of the solution which contained the specified corrosion agent. The contact time with the corrosion agent was set as $60 \mathrm{~min}$. The following acids were used: hydrochloric acid, nitric acid, sulfuric acid, and acetic acid. For each acid two concentrations were tested: 1 and $5 \mathrm{M}$. For comparison, the reference test in $1 \mathrm{M} \mathrm{KNO}_{3}$ was also prepared. The corrosion resistance studies were also carried out under galvanic exchange reaction. The experiments were performed in the same manner as in the case of 
the use of acids. Two galvanic agents were used, i.e., $\mathrm{CuCl}_{2}(1$ and $3 \mathrm{M})$ and $\mathrm{AgNO}_{3}(1$ and $5 \mathrm{M})$. The tests were performed at boiling point temperature only.

The corrosion resistance of carbon-encapsulated iron nanoparticles was also studied under active gaseous environment. A sample (120 mg) of p-CEINs was treated in a glow-discharge plasma reactor for $60 \mathrm{~min}$. The glow discharge was operated at direct current conditions and at the constant pressure of $50 \mathrm{~Pa}$ with the delivered power of $20 \mathrm{~W}$. The glow discharge was carried out in pure argon, pure oxygen, water vapor, and ammonium water vapor atmosphere. After the plasma treatment, the recovered sample $(100 \mathrm{mg})$ was shaken for $60 \mathrm{~min}$ in $50 \mathrm{~cm}^{3} 0.1 \mathrm{M} \mathrm{HCl}$.

The materials recovered after corrosion tests were weighted and analyzed. The morphological details were studied by transmission electron microscopy, while the phase composition was evaluated by powder X-ray diffraction ( $\mathrm{Cu} \mathrm{k}$-alpha radiation was used). Raman spectra were acquired using a dispersive spectrometer equipped with a 515-nm Ar-ion excitation laser. Magnetic hysteresis loops were measured using a vibrating magnetometer at room temperature. The measured magnetic moments were referred to the total mass of the studied sample. Thermogravimetric (TGA) studies were carried out under $\mathrm{N}_{2}-\mathrm{O}_{2}(60 / 40)$ atmosphere with the heating rate $10{ }^{\circ} \mathrm{C} \mathrm{min}^{-1}$. The content of $\mathrm{Fe}$ in carbon-encapsulated iron nanoparticles was evaluated from TGA curves according to the procedure described elsewhere [21].

\section{Results and discussion}

\section{Characterization of carbon-encapsulated iron nanoparticles}

Figure 1 shows selected TEM images of raw and purified carbon-encapsulated iron nanoparticles. The r-CEINs sample consists of regular (in shape) nanoparticles with the diameter between 10 and $100 \mathrm{~nm}$. A fraction of the nanoparticles is non-encapsulated or partially encapsulated in carbon. The purification in hydrochloric acid removes these nanoparticles. After purification, the product comprises of carbon-encapsulated nanoparticles, and additionally some amount of empty hollow carbon nano-onions can be found (Fig. 1b). The TGA curves
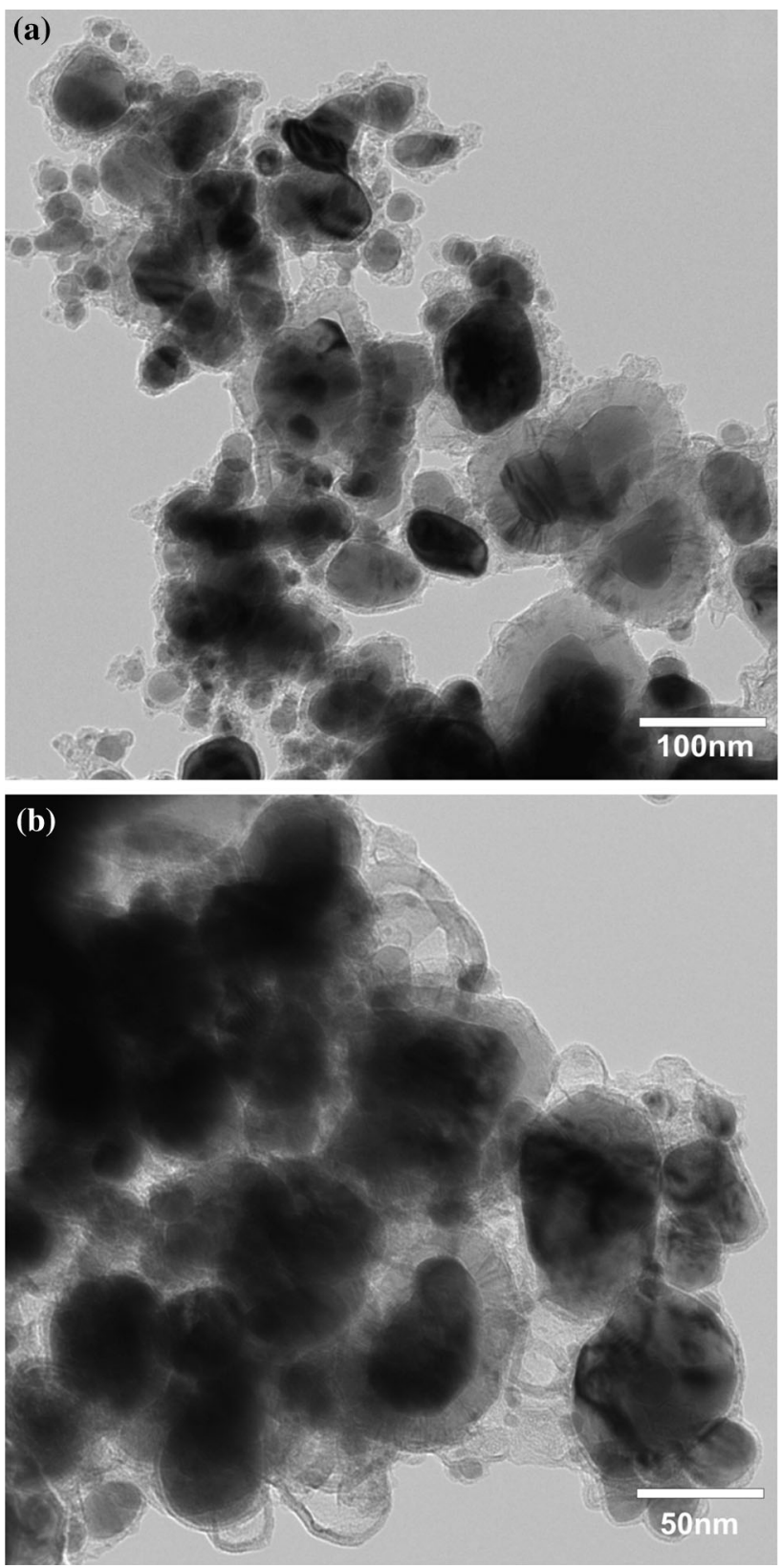

Figure 1 Selected TEM images of raw (a) and purified (b) carbon-encapsulated iron nanoparticles.

of raw and purified CEINs are shown in Fig. 2. The each curve can be splitted into three segments. The first segment (from room temperature to ca. $120-150{ }^{\circ} \mathrm{C}$ ) is accompanied by a petite mass loss (less than $1 \mathrm{wt} \%$ ) and results from the desorption of moisture and adsorbed gases. The mass gain in the second segment $\left(200-400{ }^{\circ} \mathrm{C}\right)$ is related to the oxidation of Fe nanoparticles which are accessible to oxygen. Please note that the purification of the raw material does not completely eliminate the iron 

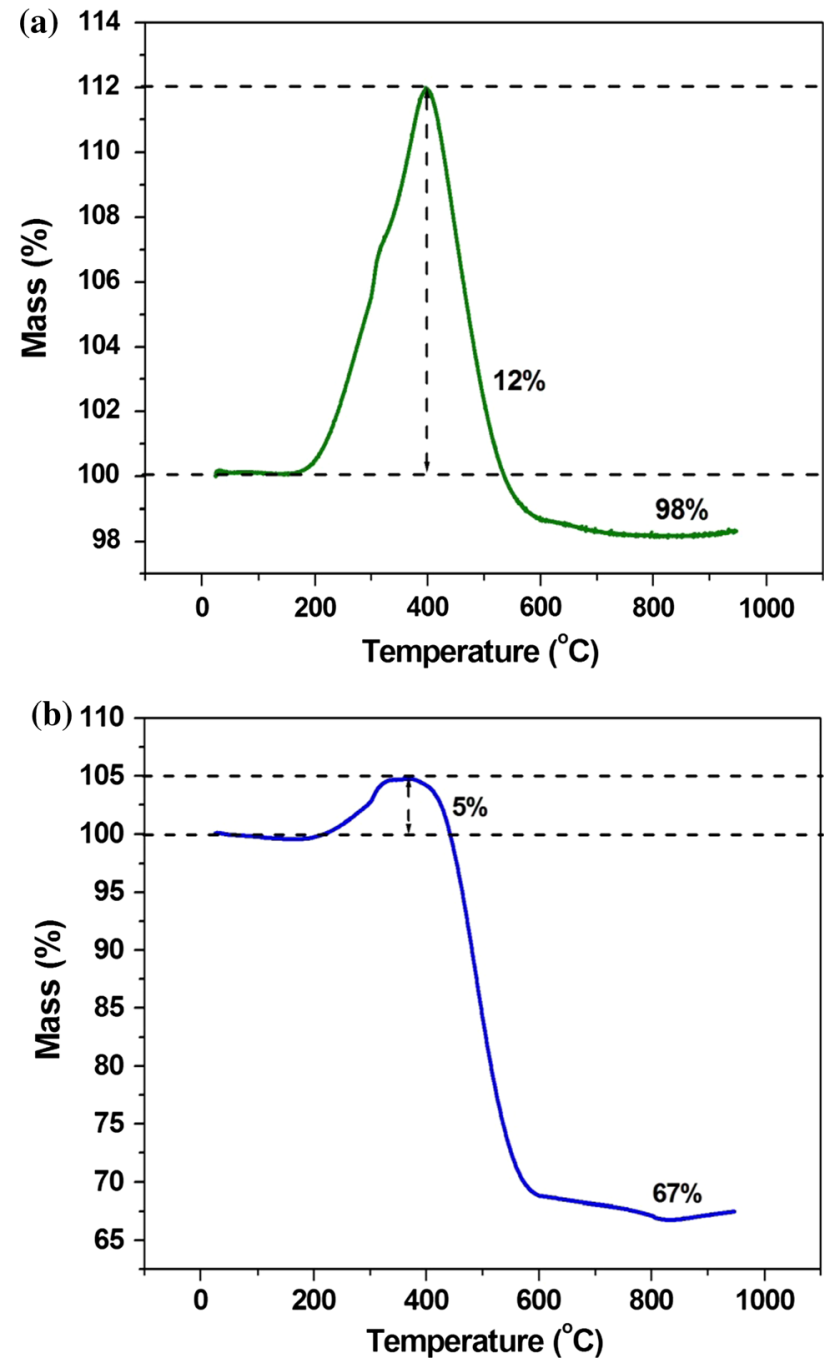

Figure 2 TGA curves of raw (a) and purified (b) carbonencapsulated iron nanoparticles.

nanoparticles which are accessible to oxygen, because of the observed mass gain ( $5 \mathrm{wt} \%$ ) in the sample of p-CEINs (Fig. 2b). This finding proves that the purification procedure results in CEINs in which some carbon coatings are plausibly more defected and are permeable to oxygen. The third segment which appears between 400 and $650{ }^{\circ} \mathrm{C}$ corresponds to the gasification of carbon phase and oxidation of encapsulated iron nanoparticles. The final mass is the mass of the as-formed iron oxide, and its value is used to evaluated the total Fe content in CEINs [21]. The Fe content in the raw and purified CEINs is 69 and $47 \mathrm{wt} \%$, respectively. The r-CEINs and p-CEINs have the same qualitative phase composition (the powder X-ray diffraction patterns are shown in Figure S1). They comprise of four crystalline phases: disordered graphite, bcc $\mathrm{Fe}, \mathrm{Fe}_{3} \mathrm{C}$, and fcc $\mathrm{Fe}-\mathrm{C}$ (austenite). Both raw and purified CEINs have ferromagnetic properties with the coercive field ca. 330 Oe (magnetic hysteresis loops are shown in Figure S2). The saturation magnetization of r-CEINs and p-CEINs is 110 and $68 \mathrm{emu} \mathrm{g}^{-1}$, respectively.

\section{Corrosion resistance studies in mineral and organic acids}

Figure 3 shows the Fe content in carbon-encapsulated iron nanoparticles subjected to corrosion tests in mineral and organic acids. The $\mathrm{Fe}$ content (45-47 wt\%) in CEINs treated with corrosion agents at room temperature is nearly the same for most of the samples. An exemption is seen for CEINs-5 M $\mathrm{H}_{2} \mathrm{SO}_{4}$ system and in this case the $\mathrm{Fe}$ content decreases to $32.2 \mathrm{wt} \%$. However, this decrease is not purely related to the dissolution of the pristinely encapsulated iron. The TGA curve for the sample treated in $5 \mathrm{M}$ sulfuric acid shows a relatively large
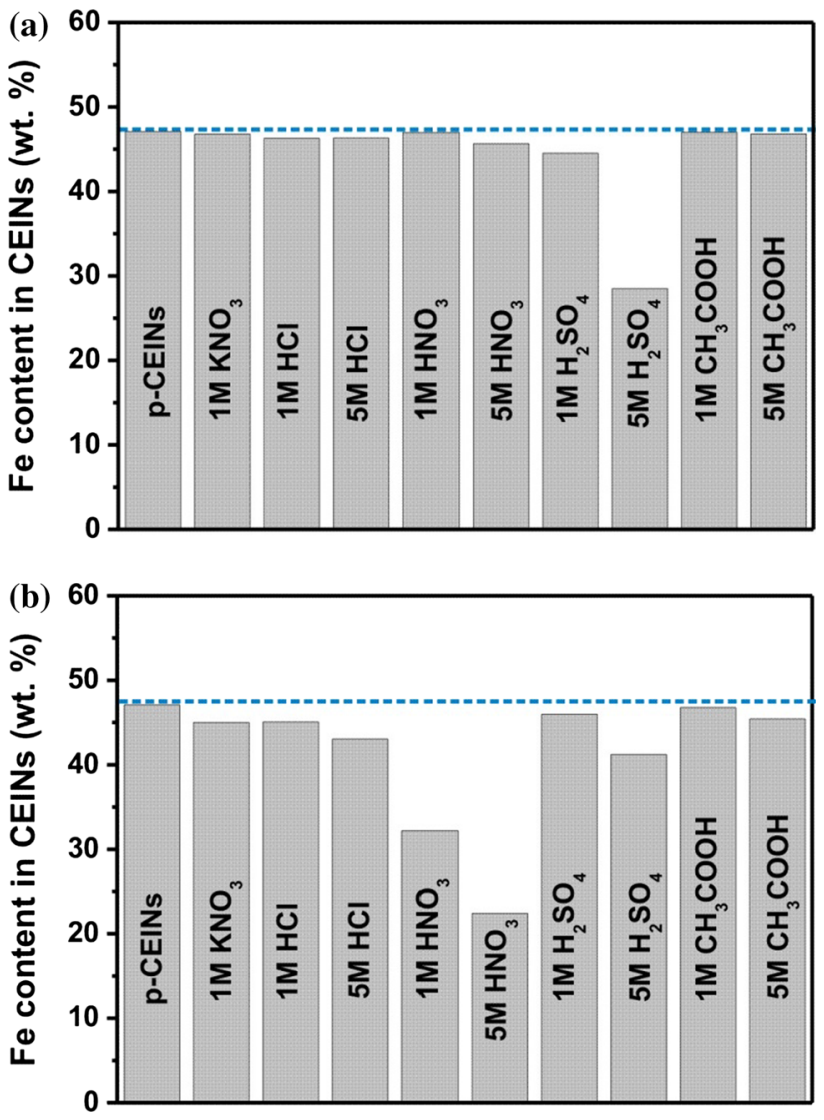

Figure $3 \mathrm{Fe}$ content in carbon-encapsulated iron nanoparticles subjected to corrosions tests in mineral and organic acids at room (a) and boiling point (b) temperature. 
mass loss (31 wt \%) in the temperature window up to $200{ }^{\circ} \mathrm{C}$ (Fig. 4). The mass loss of $16 \mathrm{wt} \%$ is related to the thermal desorption of moisture and adsorbed gases. The further loss (15 wt\%), which occurs at higher temperature (and is completed at $200{ }^{\circ} \mathrm{C}$ ) is plausibly associated with the evolution of sulfur oxides. The presence of sulfonic functionalities on the surface of CEINs treated in $5 \mathrm{M}$ sulfuric acid was confirmed by infrared spectroscopy (Fig. 5). This finding is consistent with the results published by Gomes et al. who treated activated carbon with sulfuric acid [22]. Interestingly, the corrosion test in $1 \mathrm{M}$ sulfuric acid does not result in the formation of surface functionalities. These statements are supported by a marginal mass loss (Fig. 4) and the absence of
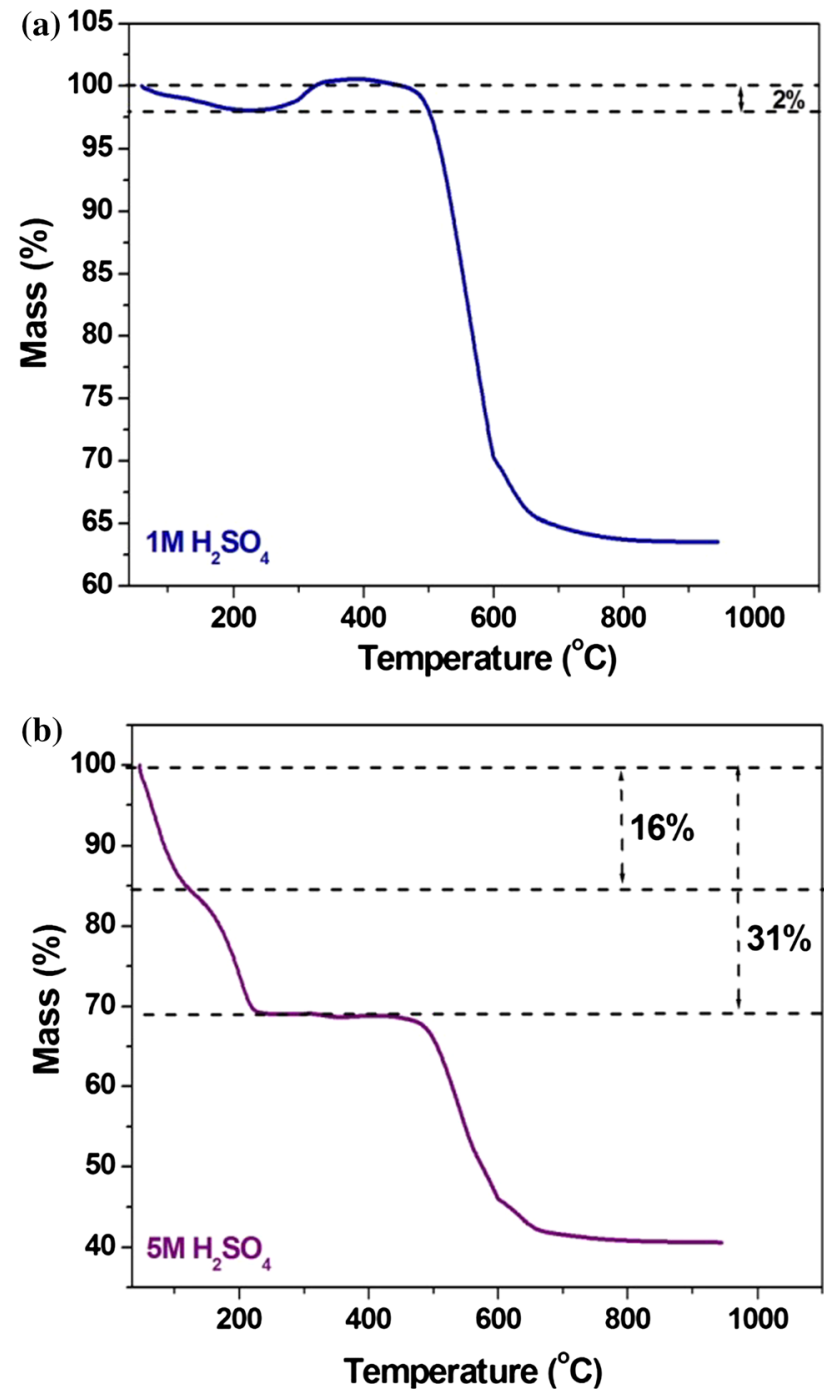

Figure 4 Thermogravimetric curves of carbon-encapsulated iron nanoparticles subjected to corrosion tests in sulfuric acid at room temperature.

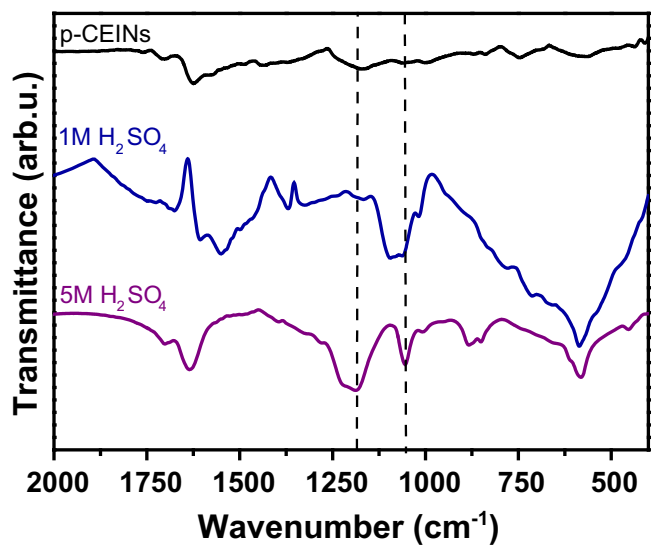

Figure 5 FTIR spectra of carbon-encapsulated iron nanoparticles subjected to corrosion tests in sulfuric acid at room temperature. The region corresponding to sulfonic group is labeled by dashed lines.

typical bands on the IR spectrum (Fig. 5). When the contribution of adsorbed water vapor and sulfur oxides in CEINs treated in $5 \mathrm{M} \mathrm{H}_{2} \mathrm{SO}_{4}$ will be excluded, then the Fe content in this sample is ca. $42 \mathrm{wt} \%$. This value is comparable to the Fe content in CEINs treated with other corrosion agents.

The $\mathrm{Fe}$ content in carbon-encapsulated iron nanoparticles subjected to the corrosion tests with non-oxidizing acids at the boiling point temperature is slightly lower (41-46 wt\%) in comparison with the tests conducted at room temperature (Fig. 3b). The samples treated in nitric acid have lower Fe content, i.e., 32.2 and $22.4 \mathrm{wt} \%$ for 1 and $5 \mathrm{M} \mathrm{HNO}_{3}$, respectively. This finding shows that hot nitric acid has a high corrosion potential and leads to the perforation of carbon coatings in CEINs with subsequent dissolution of pristinely encapsulated Fe nanoparticles.

The variation of the Fe content is a parameter which describes the corrosion resistance in a semiquantitative way. A more detailed quantification of the corrosion resistance can be evaluated when one takes into account the relative amount of iron (RAI) which was released from carbon-encapsulated iron nanoparticles after the corrosion test. This parameter is calculated as follows: $\mathrm{RAI}=\left(\frac{A_{0}-A_{\mathrm{k}}}{A_{0}}\right) \times 100 \%$, where $A_{0}$ is the content of Fe in pristine CEINs subjected to the corrosion test and $A_{\mathrm{k}}$ is the content of Fe in the sample recovered after the corrosion test, respectively. The RAI was calculated using the Fe contents from the TGA analysis. Figure 6a shows the values of RAI parameter for CEINs after corrosion tests at room temperature. The use of non-oxidative 

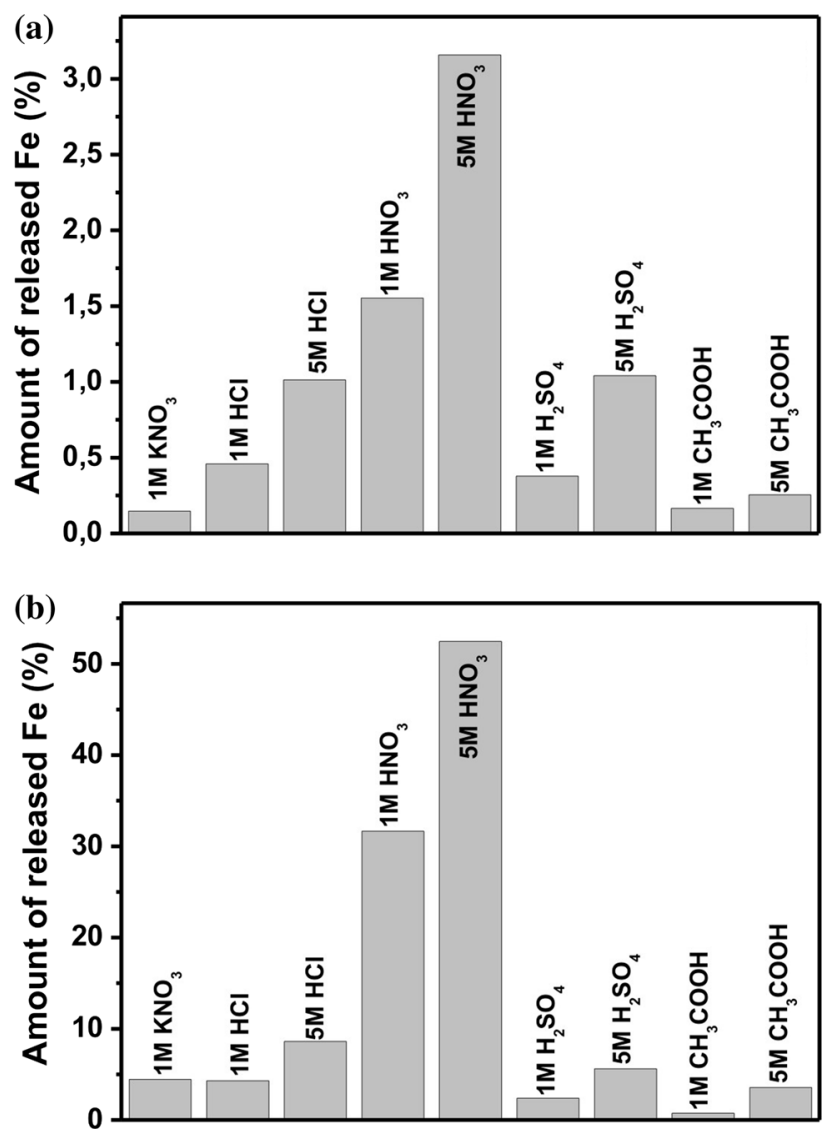

Figure 6 Relative amount of Fe released from purified carbonencapsulated iron nanoparticles subjected to corrosions tests with mineral and organic acids at room (a) and boiling (b) temperature.

mineral and organic acids results in a very petite release of pristinely encapsulated iron. The RAI parameter in this case does not exceed $1.2 \%$. The corrosion test conducted in nitric acid, which has substantially higher oxidative potential, is accompanied by greater losses of Fe content. The use of $5 \mathrm{M}$ $\mathrm{HNO}_{3}$ slightly perforates the carbon coating in CEINs and decrease the Fe content of ca. 3.2\%. The corrosion resistance changes completely for the test conducted at boiling point. The RAI value for CEINs treated in neutral electrolyte $\left(\mathrm{KNO}_{3}\right)$ as well as for acetic, sulfuric and hydrochloric (only $1 \mathrm{M}$ ) acids are on a similar level. In this case the RAI parameter is not greater than $5 \%$. The treatment in hot $5 \mathrm{M}$ hydrochloric acid use causes a larger release of encapsulated iron (7.5\%), which is ca. 9 times higher in comparison with the test at room temperature. The corrosion potential of hot nitric acid is the highest. The test performed in hot $1 \mathrm{M} \mathrm{HNO}_{3}$ results in the removal of $31.6 \%$, while the treatment in $5 \mathrm{M}$ nitric acid removes more than $52 \%$ of pristinely encapsulated iron.

The treatment in various corrosion agents affects the morphology of carbon-encapsulated iron nanoparticles. The morphological features were not changed in the samples in which the RAI parameter was lower than 5\%. The CEIN samples treated in hot nitric acid have larger amount of empty hollow carbon nano-onions (Fig. 7a, b). This finding demonstrates that this corrosion agent perforates the carbon coatings and dissolves pristinely encapsulated $\mathrm{Fe}$ particles. The outer diameter of empty carbon onions is similar to the diameter of CEINs in the p-CEINs sample. The carbon onions have well developed and continuous core-shell structure. This observation suggests that nitric acid leads to a very discrete perforation and the as-formed pores in the carbon coating were too small to be visualized on the TEM images.

As shown above nitric acid has the highest corrosion potential and the treatment of CEINs in this corrosion agent also affects the thermal resistance of CEINs. The TGA curves of CEINs treated in hot $\mathrm{HNO}_{3}$ are shown in Fig. 8. The curves are different in comparison with the pristine CEINs (Fig. 2). Obviously, the final mass is lower, because nitric acid perforates the carbon coatings and leaches the iron particles which are exposed to the acid. The difference is also seen in the temperature range between 200 and $400{ }^{\circ} \mathrm{C}$. In the case of the starting p-CEINs there is a mass gain in this region due to the oxidation of the Fe particles which are accessible to oxygen. The samples treated in hot nitric acid have different behavior because the mass decreases in this temperature range. The observed mass loss (8-10 wt \%) may be attributed to: (1) thermal desorption of surface acidic groups introduced after nitric acid treatment [23] and (2) gasification of carbon phase which has lower thermal stability. In order to solve this paradigm, the TGA inspection has been also conducted under inert gas atmosphere (the curves are shown in Supplementary Data Figures S9 and S11). The mass loss in CEINs treated in nitric acid in the temperature range between 200 and $400{ }^{\circ} \mathrm{C}$ is found to be 1.5 and $2.2 \%$, respectively. These values are substantially lower in comparison with the observed mass loss during the TGA inspection in oxygen. Therefore, the gasification of the carbon phase which has lower thermal stability is the dominant process over the 

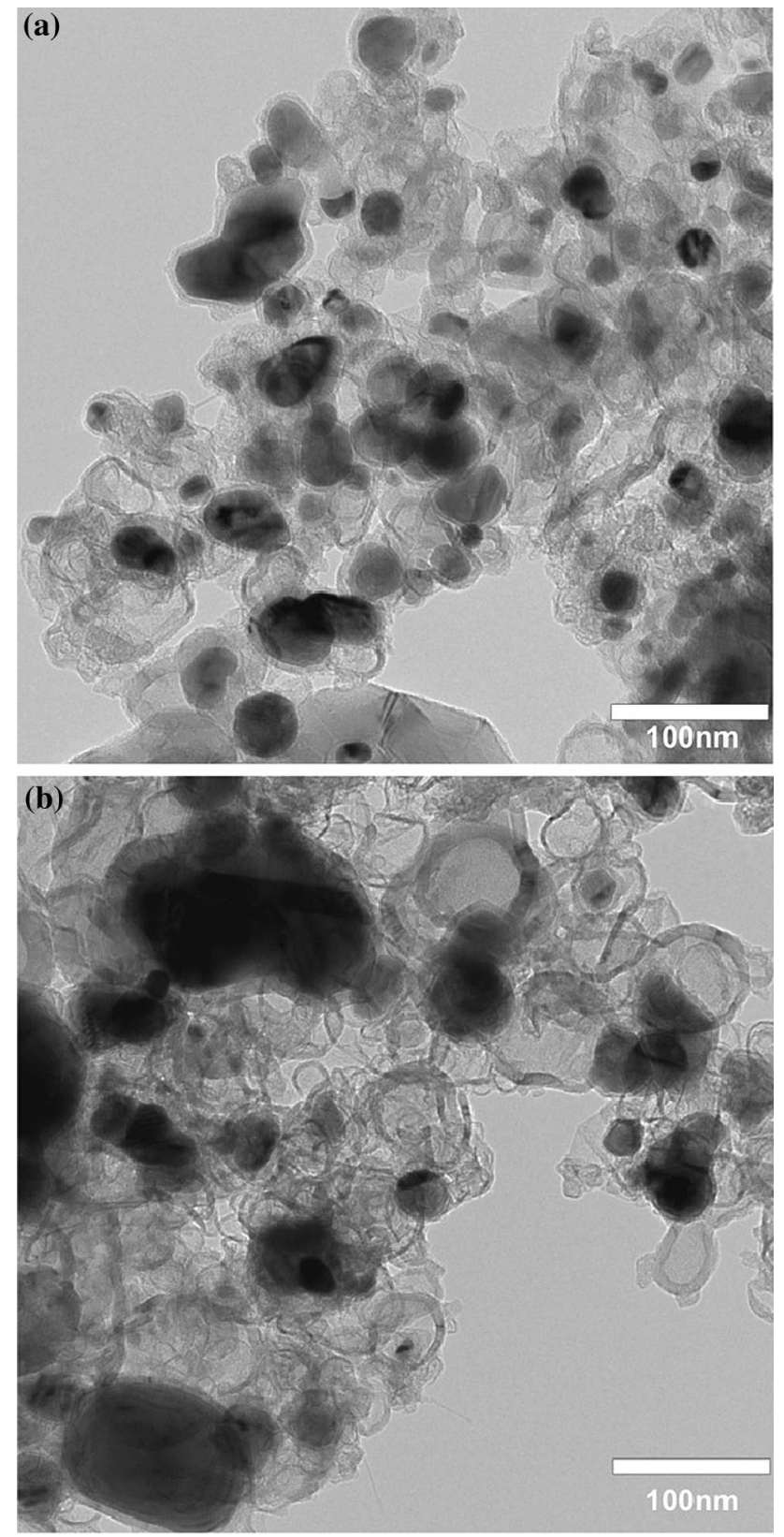

Figure 7 TEM images of purified carbon-encapsulated iron nanoparticles after corrosion tests in boiling $1 \mathrm{M} \mathrm{HNO}_{3}$ (a) and $5 \mathrm{M} \mathrm{HNO}_{3}$ (b).

thermal decomposition of surface acidic groups. This statement is also confirmed by the comparison of the position of the peak on the first derivative of the TGA curve. The "first" peak in CEINs treated in boiling $\mathrm{HNO}_{3}$ is downshifted of ca. $120-140{ }^{\circ} \mathrm{C}$ in comparison with the peak positions which correspond to the gasification of the carbon phase with higher thermal resistance $\left(477-484^{\circ} \mathrm{C}\right)$.
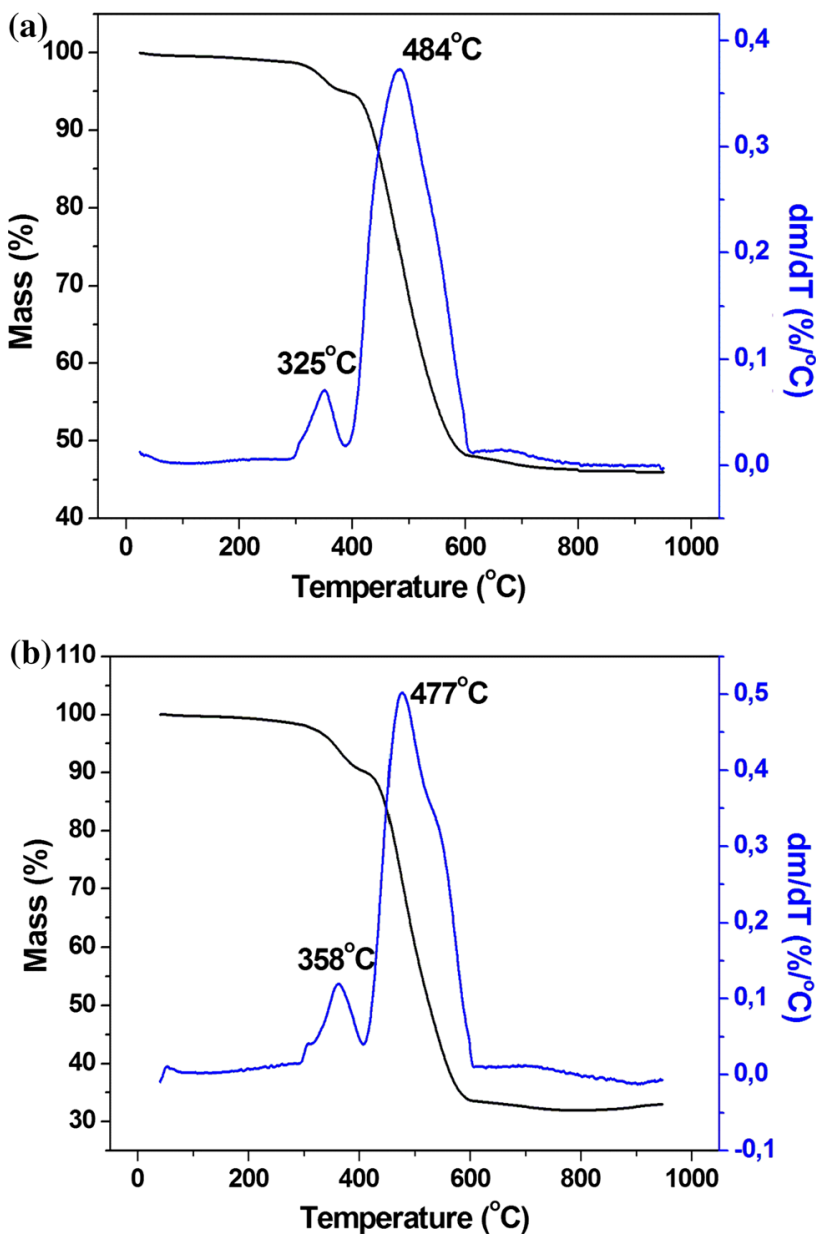

Figure 8 TGA curves of purified carbon-encapsulated iron nanoparticles after corrosion tests in boiling $1 \mathrm{M} \mathrm{HNO}_{3}$ (a) and $5 \mathrm{M} \mathrm{HNO}_{3}$ (b).

The structural features of carbon coatings were also studied by Raman spectroscopy. The Raman spectra of all studied samples are shown in Supplementary Data (Figures S24-S41). The selected Raman spectra are shown in Fig. 9. The spectra contain three major bands, i.e., the $\mathrm{G}$-band $\left(1582 \mathrm{~cm}^{-1}\right)$, the D-band $\left(1350 \mathrm{~cm}^{-1}\right)$, and the $2 \mathrm{D}$ band $\left(2700 \mathrm{~cm}^{-1}\right)$. The G/D ratio is a convenient and commonly used indicator of structural ordering in carbon materials. The G/D ratio for the pristine p-CEINs was found to be 1.8 . The G/D ratio in the samples in which the RAI parameter was lower than 5\% varied between 1.6 and 1.9. The ratio was found to be lower for samples treated with hot nitric acid, only (Fig. 9b, c). This parameter diminished to 1.3 and 0.7 for CEINs subjected to corrosion test in hot 1 and $5 \mathrm{M} \mathrm{HNO}_{3}$, respectively. This finding supports the above observations related to lowered thermal stability. Carbon 

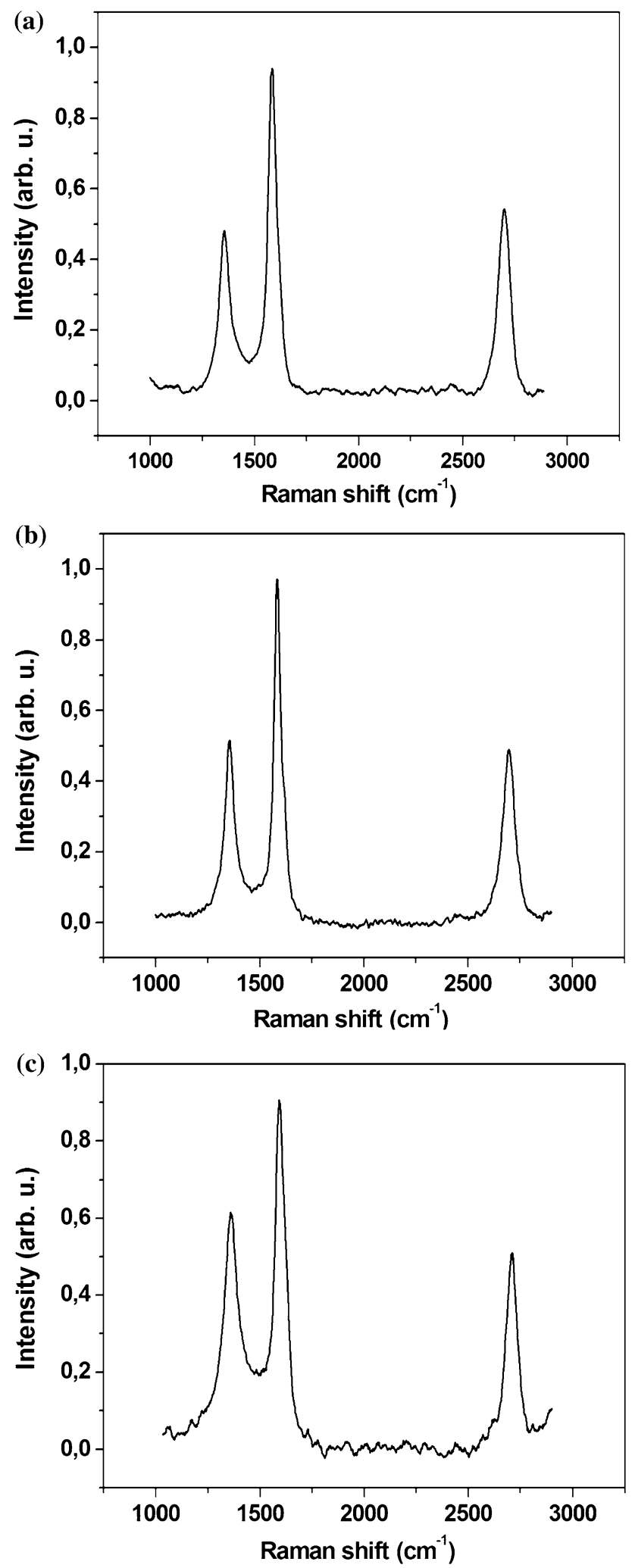

Figure 9 Raman spectra of purified carbon-encapsulated iron nanoparticles after corrosion tests in boiling $1 \mathrm{M} \mathrm{HNO}_{3}$ (a) and $5 \mathrm{M} \mathrm{HNO}_{3}$ (b). materials with lower degree of structural ordering have smaller thermal resistance to oxidation [24, 25].

The net magnetic performance, namely saturation magnetization, of carbon-encapsulated iron nanoparticles is a direct reflection of the total $\mathrm{Fe}$ content and phase composition. The observable magnetic moments in CEINs origin from ferromagnetic phases which are encapsulated in carbon, i.e., bcc Fe and $\mathrm{Fe}_{3} \mathrm{C}$. Please note that fcc $\mathrm{Fe}-\mathrm{C}$ and graphite are paramagnetic and their presence does not virtually contribute to the net magnetization. It has been described previously that the saturation magnetization in carbon-encapsulated iron nanoparticles is linearly proportional to the total Fe content [21]. As shown above, the Fe content in CEINs decreases after corrosion tests. The basic magnetic characteristics have been performed to show how the treatment in various acids changes the magnetic performance. The magnetic hysteresis loops are shown in Supplementary Data (Figures S42-S59). Figure 10 shows the relation between the saturation magnetization and the total Fe content. It is clear that the observable magnetic moment diminishes linearly with the Fe content and the corresponding changes are in agreement with the determined corrosion potential of the studied corrosion agents.

To summarize this section, one can draw a conclusion that the corrosion resistance of carbon-encapsulated iron nanoparticles is higher at room temperature. The treatment in non-oxidizing mineral and organic acids does not lead to severe perforation of carbon coatings and subsequent leaching of pristinely encapsulated Fe particles. The highest corrosion potential was found for nitric acid, and this corrosion agent, especially at boiling point, perforates the carbon shells, diminishes their structural ordering and decreases thermal stability, and finally worsens the magnetic performance of carbon-encapsulated iron nanoparticles.

\section{Galvanic corrosion}

Both of the used galvanic corrosion agents $\left(\mathrm{CuCl}_{2}\right.$ and $\mathrm{AgNO}_{3}$ ) contain the metal cation which has a high redox potential and can cause the galvanic exchange reactions with the zero-valent iron. The analysis of the TGA curves (please see Supplementary Data Figures S60-S63) shows that the final mass is higher in comparison with the p-CEINs material. This finding undoubtedly proves (in a qualitative 

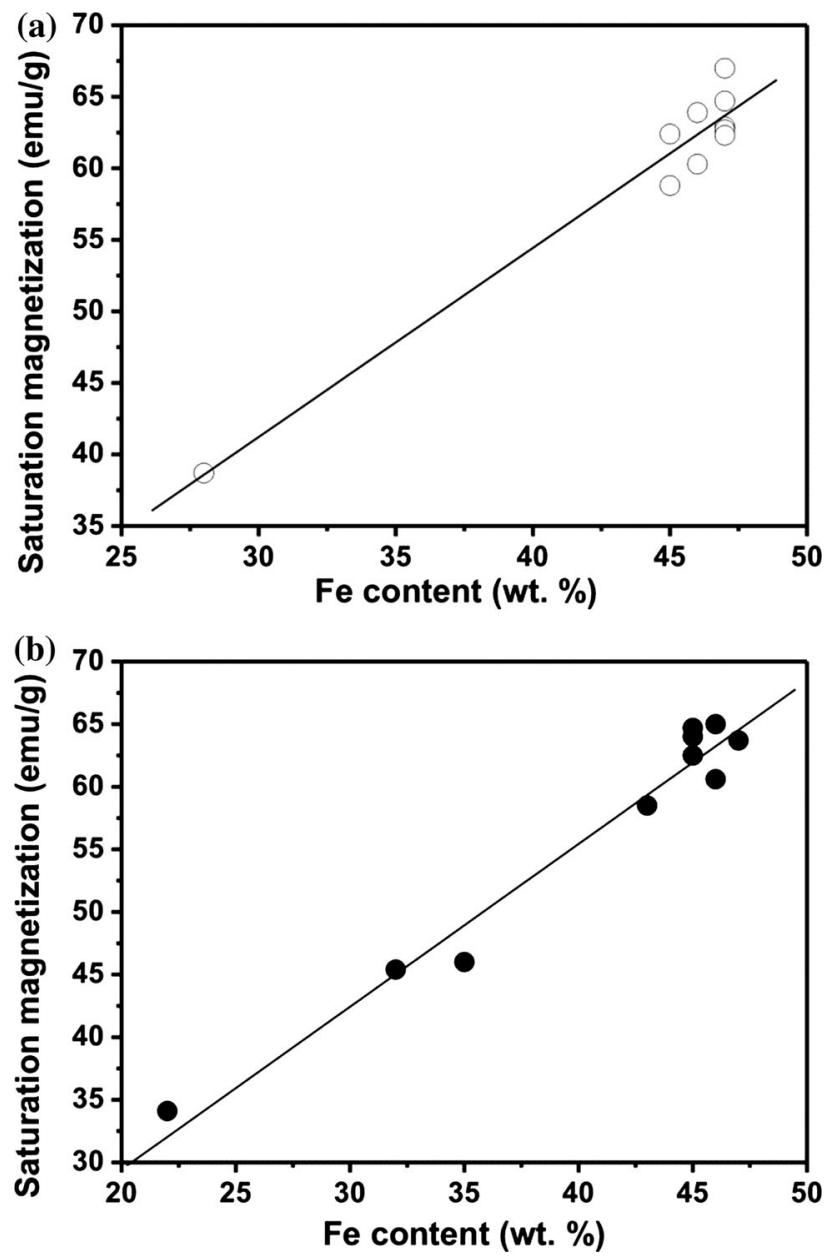

Figure 10 Relation between saturation magnetization and total Fe content for carbon-encapsulated iron nanoparticles subjected to corrosion tests with mineral and organic acids at room (a) and boiling (b) temperature.

way) that the reaction between galvanic corrosion agents and p-CEINs has occurred. This is because both copper and silver have higher atomic mass in comparison with iron. Further evidence of this reaction was tracked via powder X-ray diffraction studies (Fig. 11). The phase composition is different, i.e., new crystalline phases appear. The sample treated in copper chloride solution additionally contains $\mathrm{CuCl}$, and this observation shows that the oxidation state of copper decreased from +2 to +1 . The p-CEINs subjected to the treatment in $\mathrm{AgNO}_{3}$ solution also changed their phase composition, i.e., the sample contains additionally zero-valent silver crystallites, iron oxides and silver(I) oxide. These findings show that the galvanic exchange reaction took place and both corrosion agents oxidized the encapsulated $\mathrm{Fe}$ nanoparticles. Both $\mathrm{CuCl}$ and $\mathrm{Ag}$ are diamagnetic
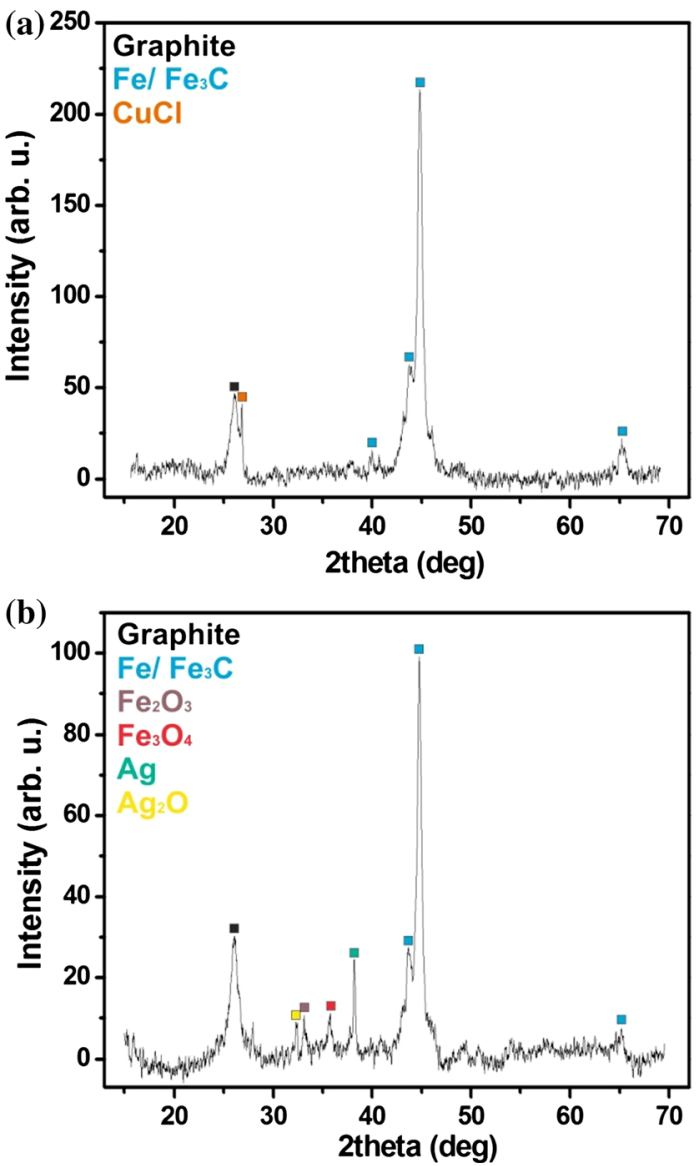

Figure $11 \mathrm{X}$-ray diffraction patterns of carbon-encapsulated iron nanoparticles subjected to galvanic corrosion tests in $3 \mathrm{M} \mathrm{CuCl}_{2}$ (a) and $5 \mathrm{M} \mathrm{AgNO}_{3}$ (b).

and their presence should decrease the net magnetic moment. In fact the measured saturation magnetization of CEINs recovered after the galvanic corrosion test is always lower $\left(42-57 \mathrm{emu}^{-1}\right)$ in comparison with pristine CEINs (Fig. 12). The decrease in magnetization is more pronounced for silver nitrate, and this corrosion factor is ca. 1.24-1.25 aggressive higher in comparison with copper(II) chloride. The magnetization also decreases with an increase of the concentration of the corrosion agent. This last statement is in agreement with the corrosion studies in acids, which also showed larger corrosion activity at higher concentration.

\section{Corrosion resistance studies in ionized gases}

The samples after plasma treatment were collected quantitatively and the mass change was monitored. In all cases the observed mass loss was between 0.9 


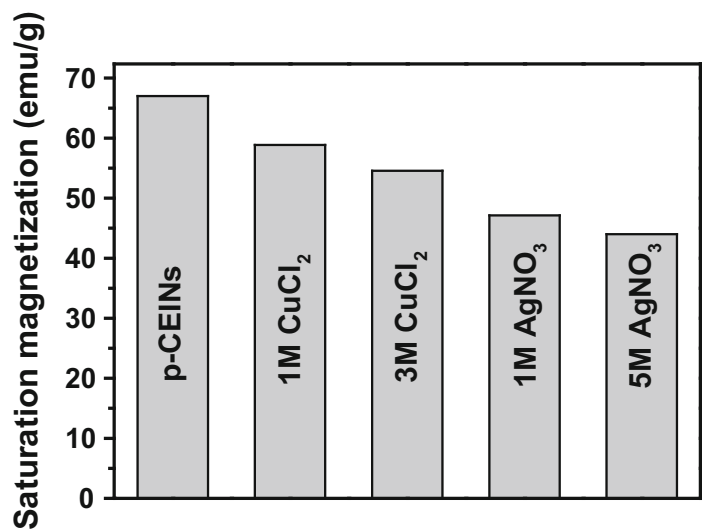

Figure 12 Saturation magnetization of carbon-encapsulated iron nanoparticles subjected to galvanic corrosion tests in $\mathrm{CuCl}_{2}$ and $\mathrm{AgNO}_{3}$.

and $2.0 \%$. This mass loss plausibly corresponds to the desorption of adsorbed gases and moisture, rather than from the gasification of the carbon coatings. Please note, that the plasma treatment was conducted at relatively low pressure $(50 \mathrm{~Pa})$ and under this condition the desorption process is favorable. The subsequent mass change of CEINs was observed after the reaction of the plasma treated samples in $0.1 \mathrm{M}$ $\mathrm{HCl}$. In this case, the mass decrease of the samples treated in $\mathrm{Ar}$ and $\mathrm{O}_{2}$ plasma was lower than 1\%. For the CEINs processed in $\mathrm{H}_{2} \mathrm{O}$ and $\mathrm{NH}_{3} / \mathrm{H}_{2} \mathrm{O}$ plasma, the mass loss was slightly higher and was found to be 2.3 and $3.4 \%$, respectively. The Fe content evaluated from the TGA curves (both after the plasma treatment and after the reaction in diluted $\mathrm{HCl}$ ) was nearly the same as in p-CEINs sample. Therefore, we have decided to determine the $\mathrm{Fe}^{3+}$ concentration in the solution recovered after the reaction in diluted $\mathrm{HCl}$. The solutions were filtered and the concentration of $\mathrm{Fe}^{3+}$ was evaluated spectrophotometrically using the thiocyanate method. Therefore, the amount of released Fe from CEINs could be estimated and it was possible to calculate the RAI parameter (in the same way as in "Corrosion resistance studies in mineral and organic acids" section). As shown in Fig. 13, the plasma treatment results in a very petite release of Fe from CEINs. The highest amount of $\mathrm{Fe}$ $(0.32 \%)$ was found in CEINs treated in oxygen plasma. Nevertheless, the observed RAI values are on a very low level and are even smaller than in CEINs treated in cold non-oxidizing acids ("Corrosion resistance studies in mineral and organic acids" section). The TEM inspection of plasma treated CEINs did not reveal any changes in their morphology. The

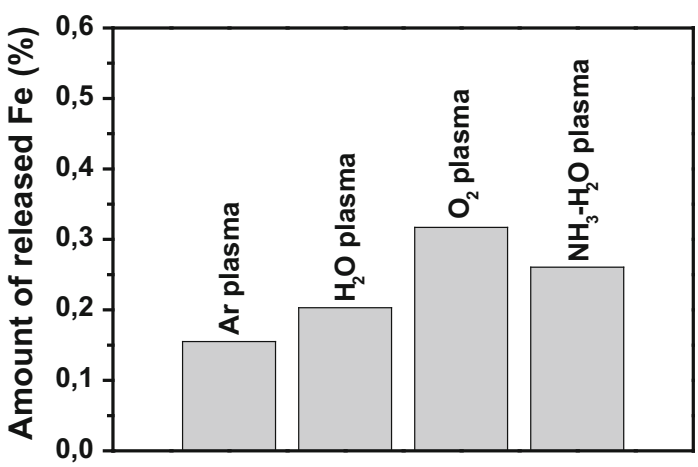

Figure 13 Amount of released Fe from purified carbon-encapsulated iron nanoparticles subjected to glow discharge.

phase composition (powder X-ray diffraction) was also not influenced by the plasma processing. Importantly, the maxima of the first derivative on the TGA curves have nearly the same position as in pristine CEINs. The plasma treatment and subsequent reaction with diluted $\mathrm{HCl}$ does not influence the magnetic performance of CEINs. The saturation magnetization of the samples was between 67 and $68 \mathrm{emu} \mathrm{g}^{-1}$ and this values are nearly identical to the saturation of pristine carbon-encapsulated iron nanoparticles $\left(67 \mathrm{emu} \mathrm{g}^{-1}\right)$. To conclude the ionized neutral (Ar) and active gases $\left(\mathrm{H}_{2} \mathrm{O}, \mathrm{NH}_{3}, \mathrm{O}_{2}\right)$ does not perforate the carbon coating in CEINs and the subsequent treatment in diluted $\mathrm{HCl}$ lead to the release of very petite amount of encapsulated iron nanoparticles.

\section{Conclusions}

The systematic studies of corrosion resistance of carbon-encapsulated iron nanoparticles have been performed. The corrosion resistance has been studied for a variety of corrosion agents, which included (1) mineral acids (sulfuric acid, nitric acid, hydrochloric acid) and organic acid (acetic acid), (2) galvanic corrosion agents [Cu(II) and $\mathrm{Ag}(\mathrm{I})]$, and (3) ionized gases $\left(\mathrm{Ar}, \mathrm{O}_{2}, \mathrm{H}_{2} \mathrm{O}, \mathrm{NH}_{3}\right.$ ). The experiments with acids were performed at room temperature and boiling point using two concentration of the corrosion agent, namely 1 and $5 \mathrm{M}$. The tests with galvanic corrosion agents were carried out at boiling point using two concentration of each agent. The experiments with ionized gases were conducted using a glow-discharge reactor and the plasma processed samples were subsequently treated in $0.1 \mathrm{M} \mathrm{HCl}$. The samples 
were thoroughly examined before and after the corrosion process and their morphological and structural features as well as magnetic performance and chemical composition was systematically monitored. It has been shown that mineral and organic non-oxidative acids do not lead to the substantial perforation of carbon coating in carbon-encapsulated iron nanoparticles. The corresponding release of Fe from carbon encapsulates does not exceeds 1.2 and $7.5 \%$ for room temperature and boiling point, respectively. Nitric acid has been found to be the most aggressive corrosion factor because the amount of released $\mathrm{Fe}$ reached 3.2 and 52\%. The treatment in nitric acid influenced also the morphological features of carbonencapsulated iron nanoparticles. The samples treated in $\mathrm{HNO}_{3}$ contained both carbon encapsulates and hollow carbon nano-onions, which originated from pristine encapsulates which were damaged by nitric acid. The corrosion activity of the studied acids was found to be: nitric acid $>$ hydrochloric acid $>$ sulfuric acid $>$ acetic acid. This order is valid for both examined concentration ( 1 and $5 \mathrm{M}$ ) and for both temperatures (room and boiling point). The galvanic corrosion studies have shown that $\mathrm{Ag}(\mathrm{I})$ have greater corrosion potential in comparison with $\mathrm{Cu}$ (II) ion. The samples recovered after galvanic reactions have broader phase composition and contained Ag- and Cu-related species along with oxidized forms of Fe. The glow-discharge tests showed that various ionized gases have very weak corrosion potential because the corresponding amount of released $\mathrm{Fe}$ was not larger than $0.3 \%$. It has been also found that the activity of the corrosion agent always rises with its concentration and is also larger at higher temperature. The release of pristinely encapsulated iron during the corrosion always diminished the magnetic performance. The highest drop of the magnetization was observed in carbon encapsulates treated in nitric acid.

This report provides systematic and accurate data on the corrosion resistance of carbon-encapsulated iron nanoparticles. The studied nanoparticles have high corrosion resistance which stems from the presence of carbon coating which tightly covers the encapsulated iron cores. The carbon coating is a barrier which protects and preserves the specific properties of carbon encapsulates. Carbon-encapsulated iron nanoparticles due to their high corrosion resistance can be used in these purposes in which the magnetic or metallic phase should not have contact with the ambient environment, e.g., (1) mobile composite adsorbents and (2) various biomedical applications (drug delivery systems, magnetic resonance imaging).

\section{Acknowledgements}

This work was supported by the National Centre for Research and Development (Poland) through the project LIDER 527/L-4/2012.

Electronic supplementary material: The online version of this article (https://doi.org/10.1007/ s10853-017-1793-z) contains supplementary material, which is available to authorized users.

Open Access This article is distributed under the terms of the Creative Commons Attribution 4.0 International License (http://creativecommons.org/ licenses/by/4.0/), which permits unrestricted use, distribution, and reproduction in any medium, provided you give appropriate credit to the original author(s) and the source, provide a link to the Creative Commons license, and indicate if changes were made.

\section{References}

[1] Ruoff RS, Lorents DC, Chan B, Malhotra R, Subramoney S (1993) Single crystal metals encapsulated in carbon nanoparticles. Science 259(5093):346-348

[2] Seshadri R, Sen R, Subbanna GN, Kannan KR, Rao CNR (1994) Iron, cobalt and nickel nanoparticles encapsulated in carbon obtained by the arc evaporation of graphite with the metals. Chem Phys Lett 231(2-3):308-313

[3] Du Y, Wang C, Toghiani H, Cai Z, Liu X, Zhang J, Yan Q (2010) Synthesis of carbon-encapsulated metal nanoparticles from wood char. For Prod J 60(6):527-533

[4] Bystrzejewski M, Károly Z, Szépvölgyi J, Kaszuwara W, Huczko A, Lange H (2009) Continuous synthesis of carbonencapsulated magnetic nanoparticles with a minimum production of amorphous carbon. Carbon 47(8):2040-2048

[5] Yin H, Gao X, Xu C, Chen P, Liu J, Zhou Q (2015) Detonation synthesis of carbon-encapsulated magnetic nanoparticles. Fuller Nanotub Carbon Nanostruct 23(7):605-611

[6] Hu Y, Zhong L, Jensen JO, Li Q (2016) Graphene layer encapsulated metal nanoparticles as a new type of non- 
precious metal catalysts for oxygen reduction. Asia-Pac J Chem Eng 11(3):382-385

[7] Kowalczyk A, Matysiak-Brynda E, Bystrzejewski M, Sutherland DS, Stojek Z, Nowicka AM (2016) Conformational control of human transferrin covalently anchored to carbon-coated iron nanoparticles in presence of a magnetic field. Acta Biomater 45:367-374

[8] Kasprzak A, Popławska M, Bystrzejewski M, Łabędź O, Grudziński IP (2015) Conjugation of polyethylenimine and its derivatives to carbon-encapsulated iron nanoparticles. RSC Adv 5(104):85556-85567

[9] Ma Y, Hu Z, Yu L, Hu Y, Yue B, Wang X, Chen Y, Lu Y, Liu $\mathrm{Y}, \mathrm{Hu} \mathrm{J}$ (2006) Chemical functionalization of magnetic carbon-encapsulated nanoparticles based on acid oxidation. J Phys Chem B 110(41):20118-20122

[10] Zlateski V, Fuhrer R, Koehler FM, Wharry S, Zeltner M, Stark WJ, Moody T, Grass RN (2014) Efficient magnetic recycling of covalently attached enzymes on carbon-coated metallic nanomagnets. Bioconjug Chem 25(4):677-684

[11] Koehler FM, Rossier M, Athanassiou EK, Limbach LK, Grass RN, Günther D, Stark WJ (2009) Magnetic EDTA: coupling heavy metal chelators to metal nanomagnets for rapid removal of cadmium, lead and copper from contaminated water. Chem Commun 32:4862-4864

[12] Taylor A, Krupskaya Y, Costa S, Oswald S, Kramer K, Fussel S, Klingeler R, Buchner B, Borowiak-Palen E, Wirth MP (2010) Functionalization of carbon encapsulated iron nanoparticles. J Nanopart Res 12:513-519

[13] Schumacher CM, Grass RN, Rossier M, Athanassiou EK, Stark WJ (2012) Physical defect formation in few layer graphene-like carbon on metals: influence of temperature, acidity and chemical functionalization. Langmuir 28:4565-4572

[14] Fernandez-Garcia MP, Gorria P, Sevilla M, Proenca MP, Boada R, Chaboy J, Fuertes AB, Blanco JA (2011) Enhanced protection of carbon-encapsulated magnetic nickel nanoparticles through a sucrose-based synthetic strategy. J Phys Chem C 115:5294-5300

[15] Schwickardi M, Olejnik S, Salabas EL, Schmidt W, Schuth F (2006) Scalable synthesis of activated carbon with superparamagnetic properties. Chem Commun 38:3987-3989
[16] Haslam GE, Chin XY, Burstein GT (2011) Passivity and electrocatalysis of nanostructured nickel encapsulated in carbon. Phys Chem Chem Phys 13:12968-12974

[17] Strachowski P, Kaszuwara W, Bystrzejewski M (2017) A novel magnetic composite adsorbent of phenolic compounds based on waste poly(ethylene terephthalate) and carbon-encapsulated magnetic nanoparticles. New J Chem 41:12617-12630

[18] Varnell JA, Tse ECM, Schulz CE, Fister TT, Haasch RT, Timoshenko J, Frenkel AI, Gewirth AA (2016) Identification of carbon-encapsulated iron nanoparticles as active species in non-precious metal oxygen reduction catalysts. Nat Commun 7:12582

[19] Kharisov BI, Rasika Dias HV, Kharissova OV, JiménezPérez VM, Olvera Pérez B, Muñoz Flores B (2012) Ironcontaining nanomaterials: synthesis, properties, and environmental applications. RSC Adv 2:9325-9358

[20] Bao Y, Wen T, Samia ACS, Khandhar A, Krishnan KM (2016) Magnetic nanoparticles: material engineering and emerging applications in lithography and biomedicine. J Mater Sci 51:513-553. https://doi.org/10.1007/s10853015-9324-2

[21] Bystrzejewski M, Łabędź O, Kaszuwara W, Huczko A, Lange H (2013) Controlling the diameter and magnetic properties of carbon-encapsulated iron nanoparticles produced by carbon arc discharge. Powder Technol 246:7-15

[22] Gomes HT, Miranda SM, Sampaio MJ, Silva AMT, Faria JL (2010) Activated carbons treated with sulphuric acid: catalysts for catalytic wet peroxide oxidation. Catal Today 151:153-158

[23] Chernyak SA, Ivanov AS, Strokova NE, Maslakov KI, Savilov SV, Lunin VV (2016) Mechanism of thermal defunctionalization of oxidized carbon nanotubes. J Phys Chem C 120:17465-17474

[24] Fronczak M, Fazekas P, Károly Z, Hamankiewicz B, Bystrzejewski M (2017) Continuous and catalyst free synthesis of graphene sheets in thermal plasma jet. Chem Eng J 322:385-396

[25] Li Z, Zhang J, Li Y, Guan Y, Feng Z, Li C (2006) Preparation and characterization of ordered mesoporous carbons on SBA-15 template. J Mater Chem 16:1350-1354 